\title{
Generalized Steiner Selections Applied to Standard Problems of Set-Valued Numerical Analysis
}

\author{
Robert Baier
}

\begin{abstract}
Generalized Steiner points and the corresponding selections for setvalued maps share interesting commutation properties with set operations which make them suitable for the set-valued numerical problems presented here. This short overview will present first applications of these selections to standard problems in this area, namely representation of convex, compact sets in $\mathbb{R}^{n}$ and set operations, set-valued integration and interpolation as well as the calculation of attainable sets of linear differential inclusions. Hereby, the convergence results are given uniformly for a dense countable representation of generalized Steiner points/selections. To achieve this aim, stronger conditions on the set-valued map $F$ have to be taken into account, e.g. the Lipschitz condition on $F$ has to be satisfied for the Demyanov distance instead of the Hausdorff distance. To establish an overview on several applications, not the strongest available results are formulated in this article.
\end{abstract}

Mathematics Subject Classification (2000). 54C65; 93B03, 93C05, 28B20.

Keywords. generalized Steiner selections, set-valued quadrature methods and interpolation, linear differential inclusions, attainable sets, Lipschitz and absolutely continuous selections, set operations.

\section{Preliminaries}

In this section, some basic notations for convex sets are introduced. $B_{r}(m)$ denotes the closed Euclidean ball with radius $r$ and center $m$ in $\mathbb{R}^{n}, B_{1}, S_{n-1}$ the unit ball resp. sphere, $\|\cdot\|$ the Euclidean norm in $\mathbb{R}^{n}$ and $\mathcal{K}_{c}\left(\mathbb{R}^{n}\right)$ the set of all convex, compact, nonempty subsets of $\mathbb{R}^{n} . \delta^{*}(l, C)$ and $Y(l, C)$ are the support function resp. the supporting face of $C \in \mathcal{K}_{c}\left(\mathbb{R}^{n}\right)$ in direction $l \in \mathbb{R}^{n}$, where $Y(l, C)$ coincides with the subdifferential of the support function. Unique supporting points are denoted by $y(l, C)$. 
In this paragraph, some well-known set operations are briefly recalled. The Minkowski sum of two sets $C, D \in \mathcal{K}_{c}\left(\mathbb{R}^{n}\right)$, the scalar multiplication with $\lambda \in \mathbb{R}$ and the image under a linear matrix $A \in \mathbb{R}^{p \times n}$ are defined as usual:

$$
C+D=\bigcup_{\substack{c \in C \\ d \in D}}\{c+d\}, \quad \lambda C=\bigcup_{c \in C}\{\lambda c\} \text { and } A C=\bigcup_{c \in C}\{A c\} .
$$

The Demyanov difference from $[16,8]$ is defined as

$$
C \cdot D=\overline{\mathrm{co}} \bigcup_{l \in T_{C} \cap T_{D}}\{y(l, C)-y(l, D)\},
$$

where $T_{C} \subset S_{n-1}$ defines the set of directions $l$ with $Y(l, C)=\{y(l, C)\}$. The Demyanov distance $\mathrm{d}_{\mathrm{D}}(C, D)$ could be calculated as the maximal norm element $\|C-D\|$ of the Demyanov difference and is stronger than the Hausdorff distance. It plays a major role in this article, since it could also be expressed by the norm of the differences of generalized Steiner points.

Within the set of all Borel probability measures on the Borel $\sigma$-algebra $\mathcal{B}$ onto $B_{1}$, a smooth measure $\beta$ is defined by a density function $\theta \in \mathcal{C}^{1}\left(B_{1}\right)$ and

$$
\beta(A)=\int_{A} \beta(d p)=\int_{A} \theta(p) d p,
$$

where $A \in \mathcal{B}$ is a Borel-measurable subset of $B_{1}$. This class of measures is shortly denoted by $\mathcal{S M}$, the so-called smooth measures.

Atomic measures from $\mathcal{A M}$ are concentrated in a single point $l \in S_{n-1}$, i.e.

$$
\alpha_{[l]}(A)= \begin{cases}0, & \text { if } l \notin A, A \in \mathcal{B}, \\ 1, & \text { if } l \in A, A \in \mathcal{B} .\end{cases}
$$

Measures with finite support in $S_{n-1}($ class $\mathcal{F} \mathcal{M})$ are convex combination of measures in $\mathcal{A M}$ (cf. [4]). $\mathcal{C} \mathcal{M}$ denotes either the family of measures $\mathcal{A M}$ or $\mathcal{F} \mathcal{M}$.

Generalized Steiner points and selections are introduced for smooth measures in $\mathcal{S M}$ by Dentcheva in $[9,10,11]$. They are generalization of the well-known Steiner center $\operatorname{St}(U)$ (take the smooth measure with uniform density in the next definition) as mentioned in [9], cf. also the references given therein.

Definition 1.1. The generalized Steiner (GS-) point of a set $C \in \mathcal{K}_{c}\left(\mathbb{R}^{n}\right)$ for a measure $\gamma \in\{\mathcal{F} \mathcal{M}, \mathcal{S} \mathcal{M}\}$ is defined as

$$
\operatorname{St}_{\gamma}(C):=\int_{B_{1}} \operatorname{St}(Y(p, C)) \gamma(d p) .
$$

Definition 1.1 equals the definition given in [9] (cf. [4, Lemma 3.3]), where the norm-minimal element of $Y(p, C)$ is used instead of the Steiner center. However, the definition above from [4] generalizes the GS-point from smooth measures to measures with finite support. For atomic measures $\alpha_{[l]}, \alpha_{[\xi]} \in \mathcal{A M}$ with $l, \xi \in S_{n-1}$ and $\lambda \in[0,1]$, the following formulas (cf. [4, Lemma 3.3]) apply for the GS-point:

$$
\operatorname{St}_{\alpha_{[l]}}(C)=\operatorname{St}(Y(l, C)), \quad \operatorname{St}_{\lambda \alpha_{[l]}+(1-\lambda) \alpha_{[\xi]}}(C)=\lambda \operatorname{St}_{\alpha_{[l]}}(C)+(1-\lambda) \operatorname{St}_{\alpha_{[\xi]}}(C)
$$


For simpler notation, we set

$$
\operatorname{St}_{\alpha_{[l]}}(C)= \begin{cases}\operatorname{St}(C), & \text { if } l=0 \mathbb{R}_{\mathbb{R}^{n}}, \\ \operatorname{St}_{\alpha_{[\eta]}}(C), & \text { if } l \neq 0_{\mathbb{R}^{n}} \text { and } \eta=\frac{1}{\|l\|} \cdot l .\end{cases}
$$

\section{Representation and Arithmetics of Sets}

GS-points form a dense, non-minimal representation of a convex compact set, i.e.

$$
C=\overline{\bigcup_{\alpha \in \mathcal{F} \mathcal{M}}\left\{\operatorname{St}_{\alpha}(C)\right\}}=\overline{\bigcup_{\beta \in \mathcal{S} \mathcal{M}}\left\{\operatorname{St}_{\beta}(C)\right\}}
$$

(see [9, Lemma 5.4] for measures in $\mathcal{S} \mathcal{M}$ resp. [4, Corollary 3.5] for the class $\mathcal{F} \mathcal{M}$ ).

Proposition 2.1. For $C \in \mathcal{K}_{c}\left(\mathbb{R}^{n}\right)$, there exists a sequence $\left(\beta_{m}\right)_{m \in \mathbb{N}} \subset \mathcal{S M}$ with

$$
C=\overline{\bigcup_{m \in \mathbb{N}}\left\{\operatorname{St}_{\beta_{m}}(C)\right\}} \text {. }
$$

The sequence of measures could also be chosen from $\mathcal{F M}$.

Proof. This follows for smooth measures from [10, Theorem 3.4]. (2.1) allows to choose a measure $\alpha_{m, N} \in \mathcal{F} \mathcal{M}$ for $N \in \mathbb{N}$ and each $m \in \mathbb{N}$ with

$$
\left\|\mathrm{St}_{\alpha_{m, N}}(C)-\mathrm{St}_{\beta_{m}}(C)\right\| \leq \frac{1}{N} .
$$

The following union gives the reprentation stated in the proposition:

$$
\bigcup_{N \in \mathbb{N}} \bigcup_{m \in \mathbb{N}}\left\{\mathrm{St}_{\alpha_{m, N}}(C)\right\}
$$

GS-points commute with the arithmetical operations for sets in $\mathcal{K}_{c}\left(\mathbb{R}^{n}\right)$, cf. [9, Remarks after Theorem 3.6] for measures in $\mathcal{S} \mathcal{M}$ resp. [4, Lemma 4.1] for $\mathcal{F} \mathcal{M}$.

Proposition 2.2. Let $C, D \in \mathcal{K}_{c}\left(\mathbb{R}^{n}\right)$ and $\gamma \in \mathcal{C M}$. Then,

$$
\begin{aligned}
\operatorname{St}_{\gamma}(\lambda C+\mu D) & =\lambda \operatorname{St}_{\gamma}(C)+\mu \operatorname{St}_{\gamma}(D) & & (\lambda, \mu \geq 0), \\
\operatorname{St}_{\gamma}(R C) & =R \operatorname{St}_{\widetilde{\gamma}}(C) & & (R \text { orthogonal matrix }) .
\end{aligned}
$$

Hereby, $\widetilde{\gamma}(B)=\gamma(R \cdot B)$ for all sets $B \in \mathcal{B}$.

Example 2.3. Let

$$
U=\operatorname{co}\left\{\left(\begin{array}{l}
2 \\
2
\end{array}\right),\left(\begin{array}{l}
0 \\
2
\end{array}\right),\left(\begin{array}{c}
-2 \\
-2
\end{array}\right),\left(\begin{array}{c}
0 \\
-2
\end{array}\right)\right\}, \quad V=\operatorname{co}\left\{\left(\begin{array}{l}
1 \\
1
\end{array}\right),\left(\begin{array}{c}
-1 \\
1
\end{array}\right),\left(\begin{array}{c}
-1 \\
-1
\end{array}\right),\left(\begin{array}{c}
1 \\
-1
\end{array}\right)\right\}
$$

and $W=U+V$. Figure 1 shows that $U$ and $V$ are represented in the left picture by 8 GS-points $\operatorname{St}_{\alpha_{\left[l^{i}\right]}}(C)$ (8 small crosses), $i=1, \ldots, 8$. By Proposition 2.2 the GS-points of $U$ and $V$ in common directions $l^{i}$ are added (see how the GS-points marked by squares resp. triangles add to form the GS-point of $W$ in the same direction in the right picture). 

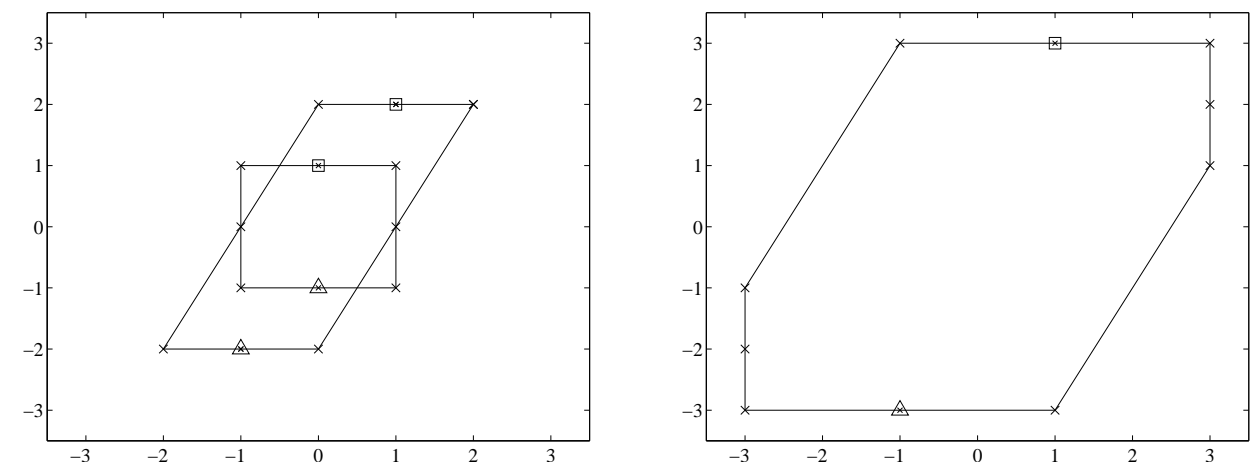

Figure 1. Minkowski sum $W$ (right) of the summands $U, V$ (left)

One could not expect that (2.2) holds for a general matrix $R \in \mathbb{R}^{n \times n}$. Nevertheless, this property could be fulfilled for special classes of sets.

Definition 2.4. The tuple $(M, U)$ with $M \in \mathbb{R}^{n \times m}$ and $U \subset \mathbb{R}^{m}$ fulfills the GSCLproperty (commutation of GS-points under linear maps), if

$$
\operatorname{St}_{\alpha_{[l]}}(M U)=M \operatorname{St}_{\alpha_{\left[M^{\top} l\right]}}(U) \text { for each } l \in S_{n-1} .
$$

Lemma 2.5. Let $M \in \mathbb{R}^{n \times m}$ and $p^{0} \in \mathbb{R}^{m}$. Then, $\left(M,\left\{p^{0}\right\}\right),\left(M, B_{1}\left(0_{\mathbb{R}^{m}}\right)\right)$ and $\left(M,[-1,1]^{m}\right)$ fulfill the GSCL-property.

Proof. Clearly, all GS-points of singletons coincide with the only element of the set, so that the case $U=\left\{p^{0}\right\}$ is simple to prove.

For a set $U$ symmetric to the origin (i.e. $U=(-1) \cdot U$ ), one has for $\eta \in S_{m-1}$ :

$$
Y(-\eta, U)=-Y(\eta, U), \quad \operatorname{St}(U)=0_{\mathbb{R}^{m}} \quad \text { and } \quad \operatorname{St}_{\alpha_{[-\eta]}}(U)=-\operatorname{St}_{\alpha_{[\eta]}}(U) .
$$

If $l \in S_{n-1}$ and $\eta:=M^{\top} l=0_{\mathbb{R}^{m}}$, then $Y\left(M^{\top} l, U\right)=U, M U=(-1) \cdot M U$ so that

$$
M \mathrm{St}_{\alpha_{\left[M^{\top} l\right]}}(U)=M \operatorname{St}(U)=0_{\mathbb{R}^{n}}=\operatorname{St}(M U)=\operatorname{St}(Y(l, M U))=\operatorname{St}_{\alpha_{[l]}}(M U) .
$$

If $\eta \neq 0_{\mathbb{R}^{m}}$, then $Y\left(\eta, B_{1}\left(0_{\mathbb{R}^{m}}\right)\right)=\left\{y\left(\eta, B_{1}\left(0_{\mathbb{R}^{m}}\right)\right)\right\}$ and $M \operatorname{St}_{\alpha_{[\eta]}}\left(B_{1}\left(0_{\mathbb{R}^{m}}\right)\right)$ equals

$$
M \operatorname{St}\left(Y\left(\eta, B_{1}\left(0_{\mathbb{R}^{m}}\right)\right)\right)=M y\left(\eta, B_{1}\left(0_{\mathbb{R}^{m}}\right)\right)=\operatorname{St}_{\alpha_{[l]}}\left(M B_{1}\left(0_{\mathbb{R}^{m}}\right)\right) .
$$

Let $v \in \mathbb{R}^{m}$. Then, $M \mathrm{St}_{\alpha_{[\eta]}}(\operatorname{co}\{-v, v\})$ coincides with

$$
\begin{aligned}
M \operatorname{St}(Y(\eta, \operatorname{co}\{-v, v\})) & =M \cdot\left\{\begin{array}{ll}
v & \text { if } \eta^{\top} v>0, \\
0_{\mathbb{R}^{m}} & \text { if } \eta^{\top} v=0, \\
-v & \text { if } \eta^{\top} v<0
\end{array}\right\}= \begin{cases}M v & \text { if } l^{\top} M v>0, \\
0_{\mathbb{R}^{n}} & \text { if } l^{\top} M v=0, \\
-M v & \text { if } l^{\top} M v<0\end{cases} \\
& =\operatorname{St}(Y(l, \operatorname{co}\{-M v, M v\}))=\mathrm{St}_{\alpha_{[l]}}(M \operatorname{co}\{-v, v\}) .
\end{aligned}
$$

The assertion follows from $[-1,1]^{m}=\sum_{i=1}^{m} \operatorname{co}\left\{-e^{i}, e^{i}\right\}$ with unit vectors $e^{i} \in \mathbb{R}^{m}$. 
An immediate consequence of Proposition 2.2 is the representation of the set operations in (1.1) with $A$ being orthoginal (cf. [4, Corollary 4.4]) as well as for the Demyanov difference/distance, cf. [4, Theorems 4.5, 4.6 and Corollary 4.8].

Theorem 2.6. Let $C, D \in \mathcal{K}_{c}\left(\mathbb{R}^{n}\right)$. Then, there exists $\left(\gamma_{m}\right)_{m \in \mathbb{N}} \subset \mathcal{C M}$ with

$$
C \cdot D=\overline{\bigcup_{m \in \mathbb{N}}\left\{\operatorname{St}_{\gamma_{m}}(C)-\mathrm{St}_{\gamma_{m}}(D)\right\}}, \quad \mathrm{d}_{D}(C, D)=\sup _{m \in \mathbb{N}}\left\|\operatorname{St}_{\gamma_{m}}(C)-\mathrm{St}_{\gamma_{m}}(D)\right\| .
$$

\section{Regularity of Set-Valued Maps}

In this paper, a set-valued map $F: I \Rightarrow \mathbb{R}^{n}$ is given with images in $\mathcal{K}_{c}\left(\mathbb{R}^{n}\right)$ and to each measure $\gamma \in \mathcal{C} \mathcal{M}$ the generalized Steiner (GS-) selection corresponds:

$$
t \mapsto \mathrm{St}_{\gamma}(F(t))
$$

It is interesting that the regularity of the set-valued map $F$ carries over to the uniform regularity of its GS-selection and vice versa, if the regularity is in some sense uniform. The first result states the Castaing representation by GS-selections characterizing the measurability of $F$ (i.e. each preimage of an open set lies in $\mathcal{B}$ ).

Theorem 3.1. Let $F: I \Rightarrow \mathbb{R}^{n}$ be measurable with images in $\mathcal{K}_{c}\left(\mathbb{R}^{n}\right)$. Then, $\operatorname{St}_{\gamma}(F(\cdot))$ is measurable for each $\gamma \in \mathcal{C} \mathcal{M}$ and there exists $\left(\gamma_{m}\right)_{m \in \mathbb{N}} \subset \mathcal{C} \mathcal{M}$ with

$$
F(t)=\overline{\bigcup_{m \in \mathbb{N}}\left\{\mathrm{St}_{\gamma_{m}}(F(t))\right\}} \quad(t \in I) .
$$

Proof. For smooth measure, this result could be found in [10, Theorem 3.4].

For atomic measures, proceed as in the proof of [10, Theorem 3.4] and choose the same measures $\left(\beta_{m}\right)_{m \in \mathbb{N}} \subset \mathcal{S} \mathcal{M}$ with densities $\left(\theta_{m}\right)_{m \in \mathbb{N}} \subset \mathcal{C}^{1}\left(B_{1}\right)$. Given an accuracy $\varepsilon>0$, each point $(t, y) \in \operatorname{graph} F$, i.e. $y \in F(t)$, could be approached as

$$
\left\|y-\operatorname{St}_{\beta_{m}}(F(t))\right\| \leq \tilde{\varepsilon}:=\frac{\varepsilon}{4 \sqrt{n}} .
$$

Apply [7, Proposition 3.4.5] to construct countable, dense sequences $\left(g_{m, k}\right)_{k \in \mathbb{N}}$ of simple, measurable functions in $L_{1}\left(B_{1}, \mathcal{B}, \beta_{m}\right)$. Let $s_{m, i}$ be the function with values of the $i$-th coordinate of $\operatorname{St}_{\beta_{m}}(F(\cdot))$. By [7, Proposition 3.4.2], there exists a simple, measurable map $h_{m, i}$ with $\left\|s_{m, i}-h_{m, i}\right\|_{L_{1}}<\tilde{\varepsilon}$. Following the proof of $[7$, Proposition 3.4.5], one could choose $g_{m, k, i}$ with $\left\|h_{m, i}-g_{m, k, i}\right\|_{L_{1}}<\tilde{\varepsilon}$. Due to the construction, $g_{m, k, i}$ is a finite sum of terms $a_{m, k, i, j} \cdot \chi_{A_{m, k, i, j}}(\cdot)$ with $a_{m, k, i, j}$ being an $\tilde{\varepsilon}$-approximation of the values $s_{m, i}$ on $A_{m, k, i, j}$. Hence, one could replace $a_{m, k, i, j}$ by $s_{m, i}\left(\xi_{m, k, i, j}\right)$ with $\xi_{m, k, i, j} \in A_{m, k, i, j}$ so that the resulting sum coincides with the measure with finite support in $\bigcup_{j=1}^{N(m, k, i)}\left\{\xi_{m, k, i, j}\right\}$. Since one could approach $(t, y) \in \operatorname{graph} F$ within accuracy $\varepsilon$, the Castaing representation is proved.

For each $l \in S_{n-1}$, the Borel measurability of the GS-selection $\operatorname{St}_{\alpha_{[l]}}(F(\cdot))$ follows from the one of marginal map $t \mapsto Y(l, F(t))$ by [6, Theorem 3.4]. Indeed, the proof of [4, Lemma 3.2] could be modified by focussing on the time $t$ instead of the direction $l$. 
Proposition 3.2 ([4, Proposition 5.1]). Let $F: I \Rightarrow \mathbb{R}^{n}$ be a set-valued map with images in $\mathcal{K}_{c}\left(\mathbb{R}^{n}\right)$. Then, $F$ is D-Lipschitz, i.e. $\mathrm{d}_{D}(F(t), F(\tau)) \leq L \cdot|t-\tau|$, if and only if for each measure $\gamma \in \mathcal{S M}$, the GS-selection $\operatorname{St}_{\gamma}(F(\cdot))$ is uniformly Lipschitz continuous with constant $L . S \mathcal{S}$ could be replaced by $\mathcal{A M}$ or $\mathcal{F} \mathcal{M}$.

E.g. the maps $F(t)=r(t) U$ with $U \in \mathcal{K}_{c}\left(\mathbb{R}^{n}\right), r(t) \geq 0$ or $A(t) B_{1}$ with $A(\cdot) \in \mathcal{C}(I), A(t)$ invertible, are D-Lipschitz. If the Lipschitz continuity of $F$ is demanded only w.r.t. the Hausdorff distance, the GS-selections for $\mathcal{S M}$ are still Lipschitz (cf. [10, Theorem 4.1]), but with constants depending on the measure.

Example 3.3 ([4, Example 5.2]). Let $I=\left[-\frac{\pi}{2}, \frac{3 \pi}{2}\right]$ and consider the set-valued map $F(t)=\operatorname{co}\left\{\left(\begin{array}{l}0 \\ 0\end{array}\right),\left(\begin{array}{l}\cos (t) \\ \sin (t)\end{array}\right)\right\}$ on $I$. Then, $F$ is Lipschitz continuous w.r.t. Hausdorff distance with constant 1 .

Consider $\theta \in \mathcal{C}^{1}\left(\mathbb{R}^{n}\right)$, the normal Dirac sequence $\left(\theta_{m}\right)_{m \in \mathbb{N}}$ from [14, Chapter 7.1C] and their measures $\beta_{m} \in \mathcal{S M}$. If $L>0$, the Lipschitz constants $L_{\beta_{m}}$ with

$$
L_{\beta_{m}}=L \cdot\left(n \cdot \max _{l \in S_{n-1}} \theta_{m}(l)+\max _{p \in B_{1}}\left\|\nabla \theta_{m}(p)\right\|\right), \quad \nabla \theta_{m}(p)=m^{n} \cdot \nabla \theta(m \cdot p),
$$

tend to $+\infty$, since the second maximum is positive and bounded uniformly in $m$.

In [4] the bounded variation of $F$ is discussed and results on the uniform bounded variation of the GS-selections are obtained. Before discussing the case of absolutely continuity, we recall the definition of the Aumann integral in [2]:

$$
\int_{I} F(t) d t=\left\{\int_{I} f(t) d t \mid f \in \mathcal{L}_{1}(I) \text { and } f \text { be a selection of } F\right\}
$$

Proposition 3.4. Let $F$ be an indefinite integral as in [1], i.e. there exists a measurable, integrably bounded $G: I \Rightarrow \mathbb{R}^{n}$ with images in $\mathcal{K}_{c}\left(\mathbb{R}^{n}\right), F_{0} \in \mathcal{K}_{c}\left(\mathbb{R}^{n}\right)$ and

$$
F(t):=F_{0}+\int_{t_{0}}^{t} G(s) d s \quad(t \in I) .
$$

Then, each GS-selection of $F$ is absolutely continuous for all $\gamma \in \mathcal{C} \mathcal{M}$ with

$$
\operatorname{St}_{\gamma}(F(t))=\operatorname{St}_{\gamma}\left(F_{0}\right)+\int_{I} \operatorname{St}_{\gamma}(G(t)) d t .
$$

Proof. The measurability of $\operatorname{St}_{\gamma}(G(\cdot))$ follows from Theorem 3.1, the integrability by the integrably boundedness of $G(\cdot)$. Equation (3.1) can be proved by Proposition 2.2 and [4, Propositions 6.2 and 6.4].

Especially, the proposition yields a dense, countable representation of the Aumann-integral by Lebesgue integrals of GS-selections with measures in $\mathcal{C} \mathcal{M}$.

\section{Set-Valued Interpolation and Quadrature Methods}

Proposition 4.1 (piecewise linear interpolation in [20]). Let $I=\left[t_{0}, T\right]$ and $F$ : $I \Rightarrow \mathbb{R}^{n}$ be a set-valued map with images in $\mathcal{K}_{c}\left(\mathbb{R}^{n}\right)$ which is D-Lipschitz with 
constant $L$. Then, the piecewise linear interpolation

$$
P_{1}(F ; t):=P_{1}(t):=\frac{t_{i+1}-t}{h} F\left(t_{i}\right)+\frac{t-t_{i}}{h} F\left(t_{i+1}\right) \quad\left(t \in\left[t_{i}, t_{i+1}\right]\right)
$$

with step-size $h=\frac{T-t_{0}}{N}, N \in \mathbb{N}$, and grid points $t_{i}=t_{0}+i h, i=0, \ldots, N$, yields

$$
\mathrm{d}_{D}\left(F(t), P_{1}(t)\right)=\sup _{m \in \mathbb{N}}\left\|\operatorname{St}_{\gamma_{m}}(F(t))-\operatorname{St}_{\gamma_{m}}\left(P_{1}(t)\right)\right\| \leq \frac{L}{4} h
$$

with a suitable sequence $\left(\gamma_{m}\right)_{m \in \mathbb{N}} \subset \mathcal{C} \mathcal{M}$.

Proof. Propositions 2.2, 3.2 and Theorem 2.6 yield:

$$
\begin{aligned}
& \mathrm{d}_{\mathrm{D}}\left(F(t), P_{1}(t)\right)=\mathrm{d}_{\mathrm{D}}\left(\frac{t_{i+1}-t}{h} F(t)+\frac{t-t_{i}}{h} F(t), \frac{t_{i+1}-t}{h} F\left(t_{i}\right)+\frac{t-t_{i}}{h} F\left(t_{i+1}\right)\right) \\
& \leq \frac{t_{i+1}-t}{h} \mathrm{~d}_{\mathrm{D}}\left(F(t), F\left(t_{i}\right)\right)+\frac{t-t_{i}}{h} \mathrm{~d}_{\mathrm{D}}\left(F(t), F\left(t_{i+1}\right)\right) \\
& \leq \frac{t_{i+1}-t}{h} \cdot L \cdot\left|t-t_{i}\right|+\frac{t-t_{i}}{h} \cdot L \cdot\left|t-t_{i+1}\right|=\frac{2 L}{h}\left(t_{i+1}-t\right)\left(t-t_{i}\right) \leq \frac{L}{4} \cdot h
\end{aligned}
$$

Hence, $F(t)$ could be densely approximated by a countable number of piecewise linear interpolants of GS-selections, since $\operatorname{St}_{\gamma_{m}}\left(P_{1}(F ; \cdot)\right)=P_{1}\left(\operatorname{St}_{\gamma_{m}}(F(\cdot)) ; \cdot\right)$.

Given some weights $b_{\mu} \geq 0$ and nodes $c_{\mu} \in[0,1], \mu=1, \ldots, s$, a set-valued quadrature formula (cf. $[12,5]$ and references therein) is given by

$$
Q(F):=\left(T-t_{0}\right) \sum_{\mu=1}^{s} b_{\mu} F\left(t_{0}+c_{\mu}\left(T-t_{0}\right)\right)
$$

for a set-valued function $F: I \Rightarrow \mathbb{R}^{n}$ with images in $\mathcal{K}_{c}\left(\mathbb{R}^{n}\right)$. The iterated version of this quadrature formula is given as

$$
Q_{N}(F):=h \sum_{i=0}^{N-1} \sum_{\mu=1}^{s} b_{\mu} F\left(t_{i}+c_{\mu} h\right)
$$

for the equi-distant step-size $h=\frac{T-t_{0}}{N}, N \in \mathbb{N}$, and nodes $t_{i}=t_{0}+i h, i=0, \ldots, N$.

In the next proposition, the assumption of bounded variation in $[4$, Proposition 6.6] is replaced by the stronger condition of Lipschitz continuity to shorten the exposition. In the proof, Propositions 2.2, 3.2, 3.4 and Theorem 2.6 are used.

Proposition 4.2. Let $F: I \Rightarrow \mathbb{R}^{n}$ have images in $\mathcal{K}_{c}\left(\mathbb{R}^{n}\right)$ and be D-Lipschitzian with constant $L$. Consider an iterated set-valued quadrature method (4.1) with $\sum_{\mu=1}^{s} b_{\mu}=1$ and $N \in \mathbb{N}$. Then, there exists $\left(\gamma_{m}\right)_{m \in \mathbb{N}} \subset \mathcal{C M}$ with

$$
\mathrm{d}_{D}\left(\int_{I} F(t) d t, Q_{N}(F)\right)=\sup _{m \in \mathbb{N}}\left\|\int_{I} \operatorname{St}_{\gamma_{m}}(F(t)) d t-Q_{N}\left(\operatorname{St}_{\gamma_{m}}(F(\cdot))\right)\right\| \leq L h
$$

Consequently, Proposition 4.2 shows that the integral of each GS-selection $\mathrm{St}_{\gamma_{m}}(F(\cdot))$ is uniformly approximated by the corresponding point-wise iterated quadrature formula of order $\mathcal{O}(h)$, since $\operatorname{St}_{\gamma_{m}}\left(Q_{N}(F(\cdot))\right)=Q_{N}\left(\operatorname{St}_{\gamma_{m}}(F(\cdot))\right)$. 

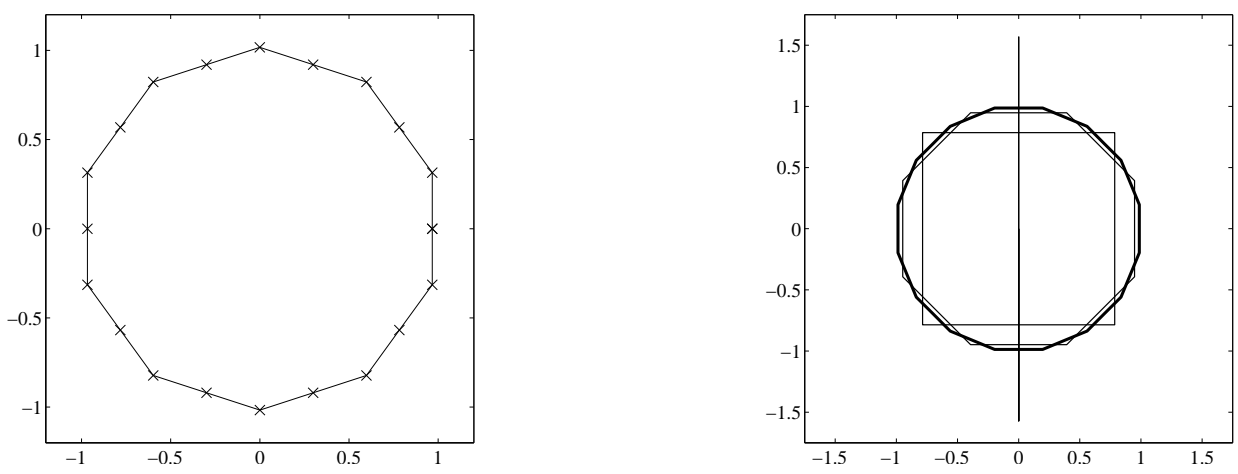

Figure 2. approximations of the Aumann integral

Example 4.3 ([19, Example before Theorem 2]). Consider $F:[0,2 \pi] \Rightarrow \mathbb{R}^{n}$ with $F(t)=\frac{1}{4} \cdot\left(\begin{array}{l}\sin (t) \\ \cos (t)\end{array}\right)[-1,1]$ and $\delta^{*}(l, F(t))=\frac{1}{4} \cdot\left|\sin (t) l_{1}+\cos (t) l_{2}\right|$ for $l \in S_{1}$.

Then, $\int_{0}^{2 \pi} F(t) d t=B_{1}$ and

$$
\mathrm{St}_{\alpha_{[l]}}(F(t))= \begin{cases}\left(\begin{array}{l}
\sin (t) \\
\cos (t)
\end{array}\right), & \text { if } \sin (t) l_{1}+\cos (t) l_{2}>0 \\
\left(\begin{array}{l}
0 \\
0
\end{array}\right), & \text { if } \sin (t) l_{1}+\cos (t) l_{2}=0 \\
-\left(\begin{array}{c}
\sin (t) \\
\cos (t)
\end{array}\right), & \text { if } \sin (t) l_{1}+\cos (t) l_{2}<0\end{cases}
$$

Clearly, $\mathrm{St}_{\alpha_{[l]}}(F(\cdot))$ has bounded variation uniformly in $l \in S_{1}$, since it is piecewise Lipschitz with maximal two jumps depending on $l$ in $I$ (the jump height is independent from $l$ ). Hence, a set-valued iterated quadrature method converges at least with order $\mathcal{O}(h)$ with the weakened form of Proposition 4.2 in [4]. Figure 2 shows the iterated Riemann sum $Q_{N}(F)=h \sum_{i=0}^{N-1} F\left(t_{i}\right)$ for $N=10$ (left picture, the GS-points are marked by crosses) and the approximating sequence of the convex hulls $Q_{N_{i}}(F)$ with $N_{i}=2^{i}, i=0,1, \ldots, 4$ (on the right, $i=4$ emphasized).

\begin{tabular}{c|rrcc||r|rrrc}
$i$ & $N$ & $M$ & $\Delta_{N}$ & $p_{N}$ & $i$ & $N$ & $M$ & $\Delta_{N}$ & $p_{N}$ \\
\hline 0 & 1 & 3 & 1.83540766 & - & 4 & 16 & 24 & 0.18109238 & 1.01091 \\
1 & 2 & 3 & 1.83540766 & 0.00000 & 5 & 32 & 40 & 0.09037697 & 1.00270 \\
2 & 4 & 8 & 0.78379807 & 1.22755 & 6 & 64 & 72 & 0.04516741 & 1.00067 \\
3 & 8 & 16 & 0.36493295 & 1.10285 & 7 & 128 & 136 & 0.02258107 & 1.00017
\end{tabular}

TABLE 1. approximate convergence order for iter. Riemann sum

Table 1 shows the approximate convergence order for the iterated Riemann sum. $N$ is the number of subintervals, $M$ is the resulting number of different GS-points, $\Delta_{N} \approx \mathrm{d}_{\mathrm{D}}\left(Q_{N}(F), \int_{I} F(t) d t\right)$ is an approximation of the Demyanov 
distance to the reference set (iterated trapezoidal rule with $N_{\text {ref }}=100000$ ) and $p_{N}$ is the estimated order of convergence which tends to the expected order 1.

\section{Linear Differential Inclusions}

Consider the linear differential inclusion (LDI) with absolutely continuous solutions $x(\cdot)$ and given integrable matrix functions $A: I \rightarrow \mathbb{R}^{n \times n}, B: I \rightarrow \mathbb{R}^{n \times m}$, a starting set $X_{0} \in \mathcal{K}_{c}\left(\mathbb{R}^{n}\right)$ and a control region $U \in \mathcal{K}_{c}\left(\mathbb{R}^{m}\right)$.

$$
\begin{aligned}
& x^{\prime}(t) \in A(t) x(t)+B(t) U \quad\left(\text { a.e. } t \in I=\left[t_{0}, T\right]\right), \\
& x\left(t_{0}\right) \in X_{0},
\end{aligned}
$$

The following representation of the attainable set $\mathcal{A}\left(T, t_{0}, X_{0}\right)$ (the set of all end points $x(T)$ of absolutely continuous solutions) is well-known and is recalled in the next lemma, cf. e.g. [17].

Lemma 5.1. Given the problem (LDI) in (5.1)-(5.2) and $l \in S_{n-1}$, the reachable set can be represented with the fundamental matrix solution $\Phi(\cdot, \cdot)$ as

$$
\begin{aligned}
\mathcal{A}\left(T, t_{0}, X_{0}\right)= & \Phi\left(T, t_{0}\right) X_{0}+\int_{I} \Phi(T, \tau) B(\tau) U d \tau \\
Y\left(l, \mathcal{A}\left(T, t_{0}, X_{0}\right)\right)= & \Phi\left(T, t_{0}\right) Y\left(\Phi\left(T, t_{0}\right)^{\top} l, X_{0}\right) \\
& +\int_{I} \Phi(T, \tau) B(\tau) Y\left(B(\tau)^{\top} \Phi(T, \tau)^{\top} l, U\right) d \tau .
\end{aligned}
$$

Proof. The second equality follows from $[15, \S 2$, Theorem 1] applied to the subdifferential $\partial \delta^{*}(l, F(t))=Y(l, F(t))$ with $F(t)=\Phi(T, t) B(t) U$.

Corollary 5.2. Given the problem (LDI) in (5.1)-(5.2), $\gamma \in \mathcal{C M}$ and $t \in I=\left[t_{0}, T\right]$, the GS-point of the reachable set evaluates as

$$
\operatorname{St}_{\gamma}\left(\mathcal{A}\left(t, t_{0}, X_{0}\right)\right)=\operatorname{St}_{\gamma}\left(\Phi\left(t, t_{0}\right) X_{0}\right)+\int_{I} \operatorname{St}_{\gamma}(\Phi(t, \tau) B(\tau) U) d \tau \quad(t \in I) .
$$

If furthermore, $X_{0}$ and $U$ are singletons, Euclidean balls or unit cubes in $\mathbb{R}^{n}$ resp. $\mathbb{R}^{m}$ and $\eta(t ; l)=\Phi\left(t, t_{0}\right)^{\top} l, \zeta(t, \tau ; l)=B(\tau)^{\top} \Phi(t, \tau)^{\top} l$, then

$$
\operatorname{St}_{\alpha_{[l]}}\left(\mathcal{A}\left(t, t_{0}, X_{0}\right)\right)=\Phi\left(t, t_{0}\right) \operatorname{St}_{\alpha_{[\eta(t ; l)]}}\left(X_{0}\right)+\int_{I} \Phi(t, \tau) B(\tau) \operatorname{St}_{\zeta(t, \tau ; l)}(U) d \tau .
$$

Proof. Clearly, Lemma 5.1 can be applied together with Lemma 2.5 , since $\left(\Phi\left(t, t_{0}\right)\right.$, $\left.X_{0}\right)$ and $(\Phi(t, \tau) B(\tau), U)$ fulfill the GSCL-property.

Equation (5.4) means that the GS-selection $u(\cdot)=\operatorname{St}_{\zeta(T, \cdot ; l)}(U)$ is the optimal control for the optimal control problem (OCP)

$$
\begin{array}{ll}
\max & l^{\top} x(T) \\
\text { s.t. } & x^{\prime}(t)=A(t) x(t)+B(t) u(t) \quad(\text { a.e. } t \in I), \\
& x\left(t_{0}\right)=\operatorname{St}_{\alpha_{[\eta(T ; l)]}}\left(X_{0}\right)
\end{array}
$$



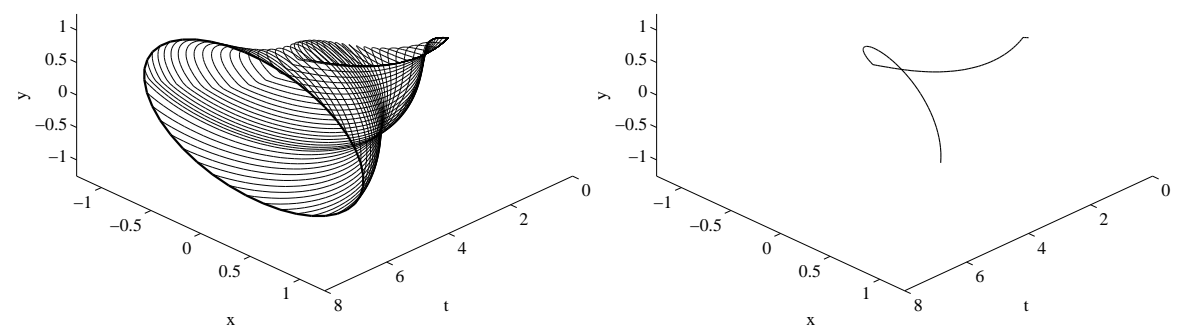

Figure 3. Euler's solutions with $N=100$ for Example 5.3

The corresponding solution $x(\cdot)$ is nothing else than an extremal solution of (LDI), where the proof was considerably simple and does not use the maximum principle. Although it should be noted that mild assumptions are available in [13] on which the strong convexity of the attainable set follows, one should observe that in any case there could not appear a multivalued situation in (5.4) as present in (5.3).

Example 5.3. Consider (LDI) on $I=[0,2 \pi]$ with

$$
A(t)=\left(\begin{array}{cc}
0 & 1 \\
-1 & 0
\end{array}\right), \quad B(t)=\frac{1}{4} \cdot\left(\begin{array}{l}
0 \\
1
\end{array}\right), \quad U=[-1,1], \quad X_{0}=\left\{0_{\mathbb{R}^{2}}\right\}
$$

The attainable set $\mathcal{A}\left(2 \pi, 0,\left\{0_{\mathbb{R}^{2}}\right\}\right)$ equals the Aumann integral in Example 4.3. Figure 3 shows 40 Euler grid functions with $N=100$ subintervals (left picture) approximating optimal solutions of the ODE in (OCP), each one corresponds to a different direction $l \in S_{n-1}$. The opening of the solution funnel is bounded by the attainable set $\mathcal{A}\left(2 \pi, 0,\left\{0_{\mathbb{R}^{2}}\right\}\right)$. In the right picture, one trajectory originating from a GS-selection is depicted which has two kinks due to jumps in the control.

\section{Conclusions}

Using the Demyanov difference in the regularity concepts of set-valued maps, error estimates which compare GS-points in common outer normals are possible (in contrast to $[20,19,12,5])$. This overview did not present the weakest versions of available results. As examples it should be mentioned that the class of sets which fulfill the GSCL-property is broader than mentioned in Lemma 2.5. One could introduce concepts of bounded variation as in [4] to establish order of convergence $\mathcal{O}(h)$ under weaker assumptions than in Propositions 4.1 and 4.2. For the generalizations for higher order in interpolation resp. quadrature methods and existence proofs of smooth dense solutions, special classes of "smooth" set-valued maps need to be studied in a forthcoming paper. Furthermore, the application of GS-points to set-valued Runge-Kutta methods (cf. $[18,3]$ ) needs further investigation. 


\section{References}

[1] Z. Artstein, On the calculus of closed set-valued functions. Indiana Univ. Math. J. 24, no. 5 (1974), 433-441.

[2] R. Aumann, Integrals of set-valued functions. J. Math. Anal. Appl. 12 (1965), 1-12.

[3] R. Baier, Selection Strategies for Set-Valued Runge-Kutta Methods. Lecture Notes in Comp. Sci. 3401 (2005), 149-157.

[4] R. Baier and E. Farkhi, Regularity and Integration of Set-Valued Maps Represented by Generalized Steiner Points. To appear in Set-Valued Anal. (2006), 22 pages.

[5] R. Baier and F. Lempio, Computing Aumann's integral. In A. Kurzhanski and V. Veliov, eds., Modeling Techniques for Uncertain Systems, Proceedings of a Conferences held in Sopron, Hungary, July 6-10, 1992. Volume 18 of Progress in Systems and Control Theory, Birkhäuser, Basel, 1994, 71-92.

[6] T. Benavides, G. Acedo and H.-K. Xu, Random fixed points of set-valued operators. Proc. Amer. Math. Soc. 124, no. 3 (1996), 831-838.

[7] D. Cohn, Measure Theory. Birkhäuser, Boston, 1980.

[8] V. Demyanov and A. Rubinov, Constructive nonsmooth analysis, volume 7 of Approximation and Optimization. Peter Lang, Frankfurt am Main, 1995.

[9] D. Dentcheva, Differentiable Selections and Castaing Representations of Multifunctions. J. Math. Anal. Appl. 223 (1998), 371-396.

[10] D. Dentcheva, Regular Castaing Representations of Multifunctions with Applications to Stochastic Programming. SIAM J. Optim. 10 (2000), 732-749.

[11] D. Dentcheva, Continuity of Multifunctions Characterized by Steiner Selections. Nonlinear Anal. 47 (2001), 1985-1996.

[12] T. Donchev and E. Farkhi, Moduli of smoothness of vector valued functions of a real variable and applications. Numer. Funct. Anal. Optim. 11, no. 5 \& 6 (1990), 497-509.

[13] H. Frankowska and C. Olech, R-convexity of the integral of the set-valued functions. In Contributions to analysis and geometry. Conference held at the Johns Hopkins University, Baltimore, Maryland, April 24-25, 1980, D. Clark, G. Pecelli and R. Sacksteder, eds., John Hopkins Univ. Press, Baltimore, MD, 1981, 117-129.

[14] F. Hirsch and G. Lacombe, Elements of Functional Analysis. Volume 192 of Graduate Texts in Mathematics. Springer, New York, 1999.

[15] A. Ioffe and V. Levin, Subdifferentials of convex functions. Trans. Moscow Math. Soc. 26 (1972), 1-72.

[16] A. Rubinov and I. Akhundov, Difference of compact sets in the sense of Demyanov and its application to non-smooth analysis. Optimization 23, no. 3 (1992), 179-188.

[17] L. Sonneborn and F. van Vleck, The bang-bang principle for linear control problems. SIAM J. Control, Ser. A, 2 (1965), 151-159.

[18] V. Veliov, Second Order Discrete Approximation to Linear Differential Inclusions. SIAM J. Numer. Anal. 29, no. 2 (1992), 439-451.

[19] V. Veliov, Discrete approximations of integrals of multivalued mappings. C. R. Acad. Bulgare Sci. 42, no. 12 (1989), 51-54.

[20] A. Vitale, Approximation of convex set-valued functions. J. Approx. Theory 26 (1979), 301-316. 
R. Baier

\section{Acknowledgment}

The author would like to thank Elza Farkhi for many suggestions and her support.

Robert Baier

University of Bayreuth

Department of Mathematics

Chair of Applied Mathematics

D-95440 Bayreuth

Germany

e-mail: robert.baier@uni-bayreuth.de 